\title{
Existence and Uniqueness of the Exponentially Stable Limit Cycle for a Class of Nonlinear Systems via Time-Domain Approach with Differential Inequality
}

\author{
Yeong-Jeu Sun, ${ }^{1}$ Yu-Biaw Wu, ${ }^{2}$ and Ching-Cheng Wang ${ }^{2}$ \\ ${ }^{1}$ Department of Electrical Engineering, I-Shou University, No.1, Sec. 1, Syuecheng Road, Dashu District, Kaohsiung City 84001, Taiwan \\ ${ }^{2}$ Institute of Manufacturing Information and Systems, National Cheng Kung University, Tainan, Taiwan \\ Correspondence should be addressed to Yeong-Jeu Sun; yjsun@isu.edu.tw
}

Received 29 November 2012; Revised 8 March 2013; Accepted 16 March 2013

Academic Editor: Samir H. Saker

Copyright ( $\odot 2013$ Yeong-Jeu Sun et al. This is an open access article distributed under the Creative Commons Attribution License, which permits unrestricted use, distribution, and reproduction in any medium, provided the original work is properly cited.

The concept of the exponentially stable limit cycle (ESLC) is introduced, and the ESLC phenomenon for a class of nonlinear systems is explored. Based on time-domain approach with differential inequality, the existence and uniqueness of the ESLC for such nonlinear systems can be guaranteed. Besides, the period of oscillation, the amplitude of oscillation, and guaranteed convergence rate can be accurately estimated. Finally, two numerical simulations are provided to illustrate the feasibility and effectiveness of the obtained result.

\section{Introduction}

Some specific nonlinear systems can offer oscillations with fixed period and fixed amplitude. Such oscillations are named limit cycles or self-oscillations, for example, an RLC electrical circuit with a nonlinear resistor, fuzzy vehicle control systems, decentralized relay systems, microwave oscillator, and the Van der Pol equation. Moreover, oscillators frequently exist in various fields of application. Recently, a wide variety of methodologies in the prediction of limit cycles have been proposed, such as describing function method, the Poincare-Bendixson theorem, piecewise-linearized methods, the Lyapunov-like approach, averaging method, analytic method, and others.

In the past, there have been some interesting developments in various limit cycle problems of nonlinear systems, but most were restricted to delay-free cases; see, for example, [1-13] and the references therein. In [13], the existence of small-amplitude limit cycles of nonlinear dynamical systems has been studied. A computational method has been proposed to obtain some new results about small limit cycles. As shown in [2], the persistent behavior of the sawtooth oscillation across different tokamaks indicates that a dynamical model based on limit cycle oscillation is consistent in contrast to the Hamiltonian models. Recently, Mendelowitz et al. have studied a system of three limit cycle oscillators which exhibits two stable steady states [8]. In [7], center conditions and bifurcation of limit cycles at the nilpotent critical point in a class of seventh degree system have been investigated. The fact that there exist 12 small amplitude limit cycles created from the three order nilpotent critical point has also been proved. Meanwhile, the driven response of time delay coupled limit cycle oscillators has been studied in [14]. In [11], the definition of the ESLC for nonlinear discrete-time systems has been firstly introduced. Based on analytic method, the existence of limit cycle for a class of nonlinear discretetime systems can be guaranteed. In addition, a uniqueness theorem of limit cycles for the Liénard system has been obtained in [12]. An example of a predator-prey system has also been given to show the application of the main theorem. In [9], the existence of limit cycles for a generalized Gausetype predator-prey model with functional and numerical responses that satisfy some general assumptions has been studied. It has been shown that the existence of a limit cycle is equivalent to the instability of the positive critical point. Besides, a model of opinion formation in a population of interacting individuals under the influence of external leaders or persuaders has been studied in [1]. The existence of 
a globally asymptotically stable periodic solution of such a model has been analytically proved in three example cases. In [3], by using the averaging method, the limit cycles for a class of quartic polynomial differential systems as well as their global shape in the plane has been investigated and discussed.

Any physical dynamic system inherently contains, more or less, some time-delay phenomena because the energy in the system propagates with a finite speed. Very often, the delay in nonlinear systems is a source of the generation of oscillation and a source of instability. These motivate us to investigate the ESLC phenomenon for a class of nonlinear time-delay systems. In this paper, based on timedomain approach with differential inequality, the existence and uniqueness of the ESLC for a class of nonlinear systems can be guaranteed. Not only the period of oscillation and amplitude of oscillation can be correctly estimated but also the guaranteed convergence rate can be accurately calculated. Finally, two numerical simulations are provided to illustrate the feasibility and effectiveness of the obtained result.

Throughout this paper, $\mathfrak{R}^{n}$ denotes the $n$-dimensional Euclidean space, $|a|$ denotes the modulus of a real number $a, N$ denotes the set of natural numbers, and $A^{T}$ denotes the transpose of the matrix $A$.

\section{Problem Formulation and Main Results}

In this paper, we consider the following nonlinear systems:

$$
\begin{aligned}
& \dot{x}_{1}(t)=-a x_{2}(t) \\
&-x_{1}(t)\left[c_{1}+c_{2} x_{i}^{2 k}(t-d)\right] \cdot\left[x_{1}^{2}(t)+x_{2}^{2}(t)-r\right], \\
& \dot{x}_{2}(t)= a x_{1}(t) \\
&-x_{2}(t)\left[c_{1}+c_{2} x_{i}^{2 k}(t-d)\right] \\
& \cdot\left[x_{1}^{2}(t)+x_{2}^{2}(t)-r\right], \\
& \forall t \geq 0,
\end{aligned}
$$

$$
x(t)=\left[\begin{array}{l}
x_{10} \\
x_{20}
\end{array}\right], \quad \forall t \in[-d, 0],
$$

where $x=\left[\begin{array}{ll}x_{1} & x_{2}\end{array}\right]^{T} \in \mathfrak{R}^{2}$ is the state vector, $a, c_{1}, c_{2}, k, i$, and $r$ are system's parameters, with $a \neq 0, c_{1}>0, c_{2} \geq 0, k \in$ $N, i \in\{1,2\}$, and $r>0$. The discrete delay argument $d$ is nonnegative. Obviously, $x=0$ is an equivalent point of system (1a) and (1b), that is, the solution of system (1a) and (1b) is given by $x(t)=0$ if $\left[\begin{array}{ll}x_{10} & x_{20}\end{array}\right]^{T}=0$. To avoid such a trivial case, in the following, we only consider the system (1a) and (1b) under the case of $\left[\begin{array}{ll}x_{10} & x_{20}\end{array}\right]^{T} \neq 0$.

The ESLC and the guaranteed convergence rate of the continuous system (1a) and (1b) are defined as follows.

Definition 1. Consider the system (1a) and (1b). The closed and bounded manifold $s(x)=0$, in the $x_{1}-x_{2}$ plane, is said to be an ESLC if there exist two positive numbers $\alpha$ and $\beta$ such that the manifold of $s(x)=0$ along the trajectories of system (1a) and (1b) satisfies the following inequality:

$$
|s(x(t))| \leq \beta \cdot \exp (-\alpha t), \quad \forall t \geq 0 .
$$

In this case, the positive number $\alpha$ is called the guaranteed convergence rate.

Now, we present the main result for the existence of the ESLC of system (1a) and (1b) as follows.

Theorem 2. All of phase trajectories of the system (1a) and (1b) tend to the ESLC $s(x)=x_{1}^{2}+x_{2}^{2}-r=0$ in the $x_{1}-x_{2}$ plane, with the guaranteed convergence rate

$$
\alpha:= \begin{cases}\infty, & \text { if } x_{10}^{2}+x_{20}^{2}=r \\ 2 c_{1} r, & \text { if } x_{10}^{2}+x_{20}^{2}>r \\ 2 c_{1}\left(x_{10}^{2}+x_{20}^{2}\right) & \text { if } x_{10}^{2}+x_{20}^{2}<r .\end{cases}
$$

Besides, the states $x_{1}(t)$ and $x_{2}(t)$ exponentially track, respectively, the trajectories

$$
\begin{aligned}
& \sqrt{r} \cos \left[a t+\tan ^{-1}\left(\frac{x_{20}}{x_{10}}\right)\right], \\
& \sqrt{r} \sin \left[a t+\tan ^{-1}\left(\frac{x_{20}}{x_{10}}\right)\right],
\end{aligned}
$$

in the time domain, with the guaranteed convergence rate $\alpha / 2$.

Proof. Define a smooth manifold $s(x)=0$ and a continuous function $\theta(x):=\tan ^{-1}\left[x_{2} / x_{1}\right]$ with $s(x)=x^{T} x-r$. Then the time derivatives of $s^{2}(x)$ and $\theta(x)$ along the trajectories of system (1a) and (1b) is given by

$$
\begin{aligned}
\frac{d s^{2}(x(t))}{d t} & =2 s(x(t)) \cdot\left(2 x_{1} \dot{x}_{1}+2 x_{2} \dot{x}_{2}\right) \\
& =-4\left[c_{1}+c_{2} x_{i}^{2 k}(t-d)\right] \cdot\left(x_{1}^{2}+x_{2}^{2}\right) s^{2}(x(t)), \\
\frac{d \theta(x(t))}{d t} & =\frac{\dot{x}_{2} x_{1}-\dot{x}_{1} x_{2}}{x_{1}^{2}+x_{2}^{2}}=a .
\end{aligned}
$$

This implies

$$
\theta(x(t))=a t+\tan ^{-1}\left(\frac{x_{20}}{x_{10}}\right) .
$$

In the following, there are three cases to discuss the trajectories of the feedback control system of (1a) and (1b).

Case 1. Consider $\left(x_{1}^{2}\left(t_{0}\right)+x_{2}^{2}\left(t_{0}\right)=r\right.$ (or equivalently; $s(x(0))=0)$ ). In this case, from (5), it can be obtained that $d s^{2}(x(t)) / d t=0$, which implies

$$
x_{1}^{2}(t)+x_{2}^{2}(t)=r, \quad \forall t \geq 0
$$


Hence we conclude that

$$
\begin{array}{cc}
x_{1}(t)=\sqrt{r} \cos \left[a t+\tan ^{-1}\left(\frac{x_{20}}{x_{10}}\right)\right], & \forall t \geq 0, \\
x_{2}(t)=\sqrt{r} \sin \left[a t+\tan ^{-1}\left(\frac{x_{20}}{x_{10}}\right)\right], & \forall t \geq 0, \\
s(x(t))=0, \quad \forall t \geq 0, &
\end{array}
$$

in view of (6) and (7).

Case 2. Consider $\left(x_{1}^{2}\left(t_{0}\right)+x_{2}^{2}\left(t_{0}\right)>r\right.$ (or equivalently; $s(x(0))>0)$ ). In this case, from (5), it can be obtained that $s^{2}(x(t))$ is a strictly decreasing function of $t$ with $s^{2}(x(t)) \geq$ $0, \forall t \geq 0$, and

$$
\begin{aligned}
\frac{d s^{2}(x(t))}{d t} & =-4\left[c_{1}+c_{2} x_{i}^{2 k}(t-d)\right]\left(x_{1}^{2}(t)+x_{2}^{2}(t)\right) s^{2}(x(t)) \\
& \leq-4 c_{1} r \cdot s^{2}(x(t)), \quad \forall t \geq 0 .
\end{aligned}
$$

Applying the differential inequality [15] with the above inequality, one has

$$
s^{2}(x(t)) \leq s^{2}(x(0)) \cdot \exp \left(-4 c_{1} r t\right), \quad \forall t \geq 0 .
$$

This implies

$$
\begin{aligned}
& |s(x(t))| \leq|s(x(0))| \cdot \exp \left(-2 c_{1} r t\right), \quad \forall t \geq 0, \\
& \quad\left|\sqrt{x_{1}^{2}(t)+x_{2}^{2}(t)}-\sqrt{r}\right|^{2} \\
& \quad \leq\left|\sqrt{x_{1}^{2}(t)+x_{2}^{2}(t)}-\sqrt{r}\right| \cdot\left|\sqrt{x_{1}^{2}(t)+x_{2}^{2}(t)}+\sqrt{r}\right| \\
& \quad=\left|x_{1}^{2}(t)+x_{2}^{2}(t)-r\right| \\
& \quad=|s(x(t))| \\
& \quad \leq|s(x(0))| \cdot \exp \left(-2 c_{1} r t\right), \quad \forall t \geq 0 .
\end{aligned}
$$

It yields

$$
\begin{aligned}
& \left|\sqrt{x_{1}^{2}(t)+x_{2}^{2}(t)}-\sqrt{r}\right| \\
& \quad \leq \sqrt{|s(x(0))|} \cdot \exp \left(-c_{1} r t\right), \quad \forall t \geq 0 .
\end{aligned}
$$

Consequently, by (6) and (12), we conclude that

$$
\begin{aligned}
\left|x_{1}(t)-\sqrt{r} \cos \left[a t+\tan ^{-1}\left(\frac{x_{20}}{x_{10}}\right)\right]\right| \\
=\mid \sqrt{x_{1}^{2}(t)+x_{2}^{2}(t)} \cos \left[a t+\tan ^{-1}\left(\frac{x_{20}}{x_{10}}\right)\right] \\
-\sqrt{r} \cos \left[a t+\tan ^{-1}\left(\frac{x_{20}}{x_{10}}\right)\right] \mid
\end{aligned}
$$

$$
\begin{aligned}
&=\left|\sqrt{x_{1}^{2}(t)+x_{2}^{2}(t)}-\sqrt{r}\right| \cdot\left|\cos \left[a t+\tan ^{-1}\left(\frac{x_{20}}{x_{10}}\right)\right]\right| \\
& \leq\left|\sqrt{x_{1}^{2}(t)+x_{2}^{2}(t)}-\sqrt{r}\right| \\
& \leq \sqrt{|s(x(0))|} \cdot \exp \left(-c_{1} r t\right), \quad \forall t \geq 0 ; \\
&\left|x_{2}(t)-\sqrt{r} \sin \left[a t+\tan ^{-1}\left(\frac{x_{20}}{x_{10}}\right)\right]\right| \\
&=\mid \sqrt{x_{1}^{2}(t)+x_{2}^{2}(t)} \sin \left[a t+\tan ^{-1}\left(\frac{x_{20}}{x_{10}}\right)\right] \\
& \quad-\sqrt{r} \sin \left[a t+\tan ^{-1}\left(\frac{x_{20}}{x_{10}}\right)\right] \mid \\
& \leq\left|\sqrt{x_{1}^{2}(t)+x_{2}^{2}(t)}-\sqrt{r}\right| \cdot\left|\sin \left[a t+\tan ^{-1}\left(\frac{x_{20}}{x_{10}}\right)\right]\right| \\
& \leq \sqrt{|s(x(0))|} \cdot \exp \left(-c_{1} r t\right), \quad \forall t \geq 0 .
\end{aligned}
$$

Case 3. Consider $\left(x_{1}^{2}\left(t_{0}\right)+x_{2}^{2}\left(t_{0}\right)<r\right.$ (or equivalently; $s(x(0))<0)$ ). In this case, from (5), it can be obtained that $s^{2}(x(t))$ is a strictly decreasing function of $t$ with $s^{2}(x(t)) \geq$ $0, \forall t \geq 0$, and

$$
\begin{aligned}
\frac{d s^{2}(x(t))}{d t} & =-4\left[c_{1}+c_{2} x_{i}^{2 k}(t-d)\right]\left(x_{1}^{2}(t)+x_{2}^{2}(t)\right) s^{2}(x(t)) \\
& \leq-4 c_{1}\left(x_{10}^{2}+x_{20}^{2}\right) s^{2}(x(t)), \quad \forall t \geq 0 .
\end{aligned}
$$

Applying the differential inequality [15] with above inequality, one has

$$
s^{2}(x(t)) \leq s^{2}\left(x\left(t_{0}\right)\right) \cdot \exp \left[-4 c_{1}\left(x_{10}^{2}+x_{20}^{2}\right) t\right], \quad \forall t \geq 0,
$$

this implies

$$
\begin{aligned}
& |s(x(t))| \leq\left|s\left(x\left(t_{0}\right)\right)\right| \cdot \exp \left[-2 c_{1}\left(x_{10}^{2}+x_{20}^{2}\right) t\right], \quad \forall t \geq 0, \\
& \left|\sqrt{x_{1}^{2}(t)+x_{2}^{2}(t)}-\sqrt{r}\right|^{2} \\
& \quad \leq\left|\sqrt{x_{1}^{2}(t)+x_{2}^{2}(t)}-\sqrt{r}\right| \cdot\left|\sqrt{x_{1}^{2}(t)+x_{2}^{2}(t)}+\sqrt{r}\right| \\
& \quad=\left|x_{1}^{2}(t)+x_{2}^{2}(t)-r\right| \\
& \quad=|s(x(t))| \\
& \quad \leq\left|s\left(x\left(t_{0}\right)\right)\right| \cdot \exp \left[-2 c_{1}\left(x_{10}^{2}+x_{20}^{2}\right) t\right], \quad \forall t \geq 0 .
\end{aligned}
$$






Figure 1: The diagram of implementation, where $C 1=C 2=1 \mu \mathrm{F}$ and $R i=100 \mathrm{k} \Omega, \forall i \in\{1,2, \ldots 24\}$.

It yields

$$
\begin{aligned}
& \left|\sqrt{x_{1}^{2}(t)+x_{2}^{2}(t)}-\sqrt{r}\right| \\
& \leq \sqrt{\left|s\left(x\left(t_{0}\right)\right)\right|} \cdot \exp \left[-c_{1}\left(x_{10}^{2}+x_{20}^{2}\right) t\right], \quad \forall t \geq 0 .
\end{aligned}
$$

Consequently, by (6) and (17), we conclude that

$$
\begin{aligned}
\left|x_{1}(t)-\sqrt{r} \cos \left[a t+\tan ^{-1}\left(\frac{x_{20}}{x_{10}}\right)\right]\right| \\
=\mid \sqrt{x_{1}^{2}(t)+x_{2}^{2}(t)} \cos \left[a t+\tan ^{-1}\left(\frac{x_{20}}{x_{10}}\right)\right] \\
-\sqrt{r} \cos \left[a t+\tan ^{-1}\left(\frac{x_{20}}{x_{10}}\right)\right] \mid
\end{aligned}
$$

$$
\begin{aligned}
&=\left|\sqrt{x_{1}^{2}(t)+x_{2}^{2}(t)}-\sqrt{r}\right| \cdot\left|\cos \left[a t+\tan ^{-1}\left(\frac{x_{20}}{x_{10}}\right)\right]\right| \\
& \leq\left|\sqrt{x_{1}^{2}(t)+x_{2}^{2}(t)}-\sqrt{r}\right| \\
& \leq \sqrt{\left|s\left(x\left(t_{0}\right)\right)\right|} \cdot \exp \left[-c_{1}\left(x_{10}^{2}+x_{20}^{2}\right) t\right], \quad \forall t \geq 0 ; \\
&\left|x_{2}(t)-\sqrt{r} \sin \left[a t+\tan ^{-1}\left(\frac{x_{20}}{x_{10}}\right)\right]\right| \\
&=\mid \sqrt{x_{1}^{2}(t)+x_{2}^{2}(t)} \sin ^{-1}\left[a t+\tan ^{-1}\left(\frac{x_{20}}{x_{10}}\right)\right] \\
& \quad-\sqrt{r} \sin \left[a t+\tan ^{-1}\left(\frac{x_{20}}{x_{10}}\right)\right] \mid
\end{aligned}
$$






$(t)$

FIGURE 2: $x_{1}(t)$ and $x_{2}(t)$ of the nonlinear system (1a) and (1b), with $a=c_{2}=k=i=1, c_{1}=2, r=4, d=0.1,\left[\begin{array}{ll}x_{10} & x_{20}\end{array}\right]^{T}=\left[\begin{array}{ll}4 & 4\end{array}\right]^{T}$.



FIGURE 3: Typical phase trajectories of the nonlinear system (1a) and (1b), with $a=c_{2}=k=i=1, c_{1}=2, \quad r=4, d=0.1,\left[x_{10} \quad x_{20}\right]^{T}=$ $\left[\begin{array}{ll}4 & 4\end{array}\right]^{T}$.

$$
\begin{aligned}
& =\left|\sqrt{x_{1}^{2}(t)+x_{2}^{2}(t)}-\sqrt{r}\right| \cdot\left|\sin \left[a t+\tan ^{-1}\left(\frac{x_{20}}{x_{10}}\right)\right]\right| \\
& \leq\left|\sqrt{x_{1}^{2}(t)+x_{2}^{2}(t)}-\sqrt{r}\right| \\
& \leq \sqrt{\left|s\left(x\left(t_{0}\right)\right)\right|} \cdot \exp \left[-c_{1}\left(x_{10}^{2}+x_{20}^{2}\right) t\right], \quad \forall t \geq 0 .
\end{aligned}
$$

This completes the proof.

Remark 3. By Theorem 2, it is clear that both of $x_{1}(t)$ and $x_{2}(t)$ can be represented as nonlinear oscillators with the amplitude $\sqrt{r}$ and the frequency $a$. Such oscillations are generally independent of the initial condition. Meanwhile, the phenomenon of limit cycle is not affected by parameter variation.
Remark 4. Based on time-domain approach with differential inequality, we may obtain the desired amplitude and the desired frequency by tuning the parameters $r$ and $a$, respectively, in view of (4). It is worthwhile to note that any member of a family of nonlinear system (1a) and (1b) not only can be regarded as nonlinear oscillator but also can be implemented by electronic circuits, as shown in Figure 1.

\section{Numerical Simulations}

Example 5. Consider the nonlinear system (1a) and (1b) with

$$
\begin{array}{r}
a=c_{2}=k=i=1, \quad c_{1}=2, \quad r=4, \\
d=0.1, \quad\left[\begin{array}{ll}
x_{10} & x_{20}
\end{array}\right]^{T}=\left[\begin{array}{ll}
4 & 4
\end{array}\right]^{T} .
\end{array}
$$




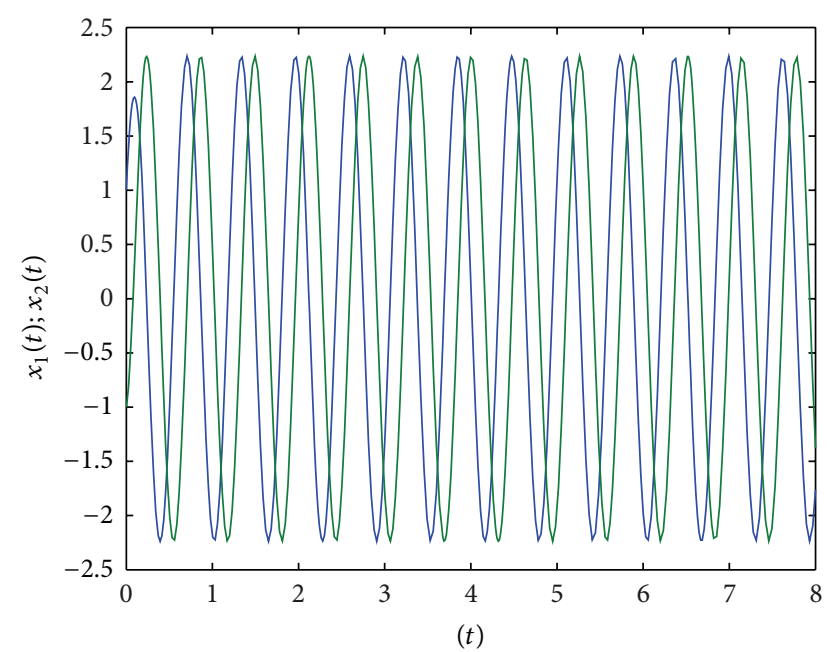

FIGURE 4: $x_{1}(t)$ and $x_{2}(t)$ of the nonlinear system (1a) and (1b), with $a=10, c_{1}=c_{2}=k=1, i=2, r=5, d=0.2,\left[\begin{array}{ll}x_{10} & x_{20}\end{array}\right]^{T}=$ $\left[\begin{array}{ll}1 & -1\end{array}\right]^{T}$.

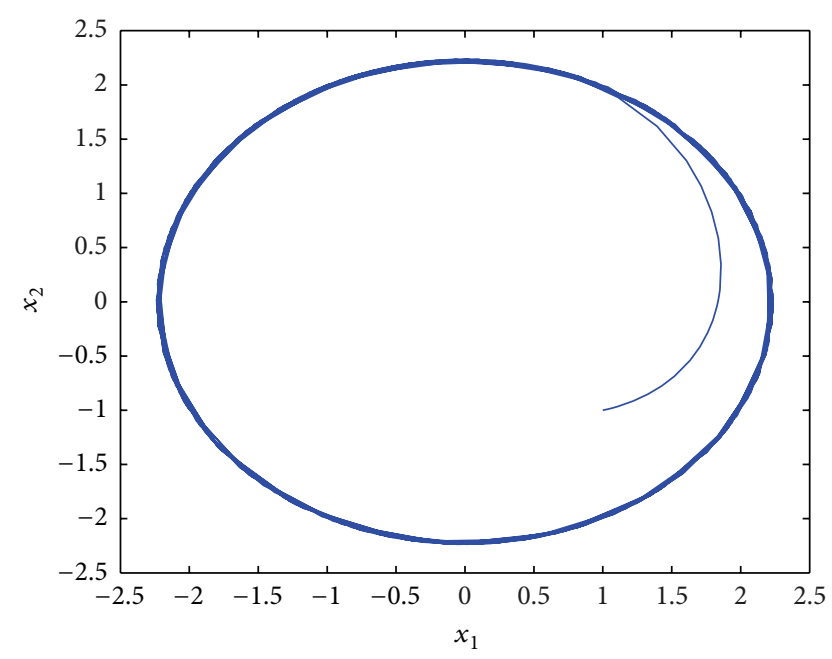

FIGURE 5: Typical phase trajectories of the nonlinear system (1a) and (1b), with $a=10, c_{1}=c_{2}=k=1, i=2, r=5, d=$ $0.2,\left[\begin{array}{ll}x_{10} & x_{20}\end{array}\right]^{T}=\left[\begin{array}{ll}1 & -1\end{array}\right]^{T}$.

By Theorem 2, we conclude that the phase trajectories of system (1a) and (1b) tend to the ESLC $s(x)=x_{1}^{2}+$ $x_{2}^{2}-4=0$ in the $x_{1}-x_{2}$ plane, with the guaranteed convergence rate $\alpha=16$. Furthermore, the states $x_{1}(t)$ and $x_{2}(t)$ exponentially track, respectively, the trajectories $2 \cos [t+(\pi / 4)]$ and $2 \sin [t+(\pi / 4)]$, in the time domain, with the guaranteed convergence rate $\alpha / 2=8$. Some state trajectories of above nonlinear system are depicted in Figures 2 and 3 . From the foregoing simulations results, it is seen that the nonlinear (1a) and (1b) with

$$
\begin{gathered}
a=c_{2}=k=i=1, \quad c_{1}=2, \quad r=4, \\
d=0.1, \quad\left[\begin{array}{ll}
x_{10} & x_{20}
\end{array}\right]^{T}=\left[\begin{array}{ll}
4 & 4
\end{array}\right]^{T},
\end{gathered}
$$

uniquely possesses the ESLC of $s(x)=x_{1}^{2}+x_{2}^{2}-4=0$.
Example 6. Consider the nonlinear system (1a) and (1b) with

$$
\begin{gathered}
a=10, \quad c_{1}=c_{2}=k=1, \quad i=2, \quad r=5, \\
d=0.2, \quad\left[\begin{array}{ll}
x_{10} & x_{20}
\end{array}\right]^{T}=\left[\begin{array}{ll}
1 & -1
\end{array}\right]^{T} .
\end{gathered}
$$

By Theorem 2, we conclude that the phase trajectories of system (1a) and (1b) tend to the ESLC $s(x)=x_{1}^{2}+x_{2}^{2}-5=0$ in the $x_{1}-x_{2}$ plane, with the guaranteed convergence rate $\alpha=4$. Furthermore, the states $x_{1}(t)$ and $x_{2}(t)$ exponentially track, respectively, the trajectories $\sqrt{5} \cos [10 t-(\pi / 4)]$ and $\sqrt{5} \sin [10 t-(\pi / 4)]$, in the time domain, with the guaranteed convergence rate $\alpha / 2=2$. Some state trajectories of above nonlinear system are depicted in Figures 4-5. From the foregoing simulations results, it is seen that the nonlinear system (1a) and (1b) with

$$
\begin{gathered}
a=10, \quad c_{1}=c_{2}=k=1, \quad i=2, \quad r=5, \\
d=0.2, \quad\left[\begin{array}{ll}
x_{10} & x_{20}
\end{array}\right]^{T}=\left[\begin{array}{ll}
1 & -1
\end{array}\right]^{T}
\end{gathered}
$$

uniquely possesses the ESLC of $s(x)=x_{1}^{2}+x_{2}^{2}-5=0$.

\section{Conclusion}

In this paper, the concept of the ESLC has been introduced and the ESLC phenomenon for a class of nonlinear systems has been explored. Based on time-domain approach with differential inequality, the existence and uniqueness of the ESLC for such nonlinear systems can be guaranteed. Besides, the period of oscillation, the amplitude of oscillation, and guaranteed convergence rate can be accurately estimated. Finally, numerical simulations have also been provided to illustrate the feasibility and effectiveness of the obtained result.

\section{Acknowledgments}

The authors thank the National Science Council of China for supporting this work under Grant No. NSC-100-2221-E-214015 .

\section{References}

[1] M. L. Bertotti and M. Delitala, "On the existence of limit cycles in opinion formation processes under time periodic influence of persuaders," Mathematical Models \& Methods in Applied Sciences, vol. 18, no. 6, pp. 913-934, 2008.

[2] M. P. Bora and D. Sarmah, "Sawtooth disruptions and limit cycle oscillations," Communications in Nonlinear Science and Numerical Simulation, vol. 13, no. 2, pp. 296-313, 2008.

[3] J. Cao, "Limit cycles of polynomial differential systems with homogeneous nonlinearities of degree 4 via the averaging method," Journal of Computational and Applied Mathematics, vol. 220, no. 1-2, pp. 624-631, 2008.

[4] Y. M. Chen and J. K. Liu, "A study of homotopy analysis method for limit cycle of van der Pol equation," Communications in Nonlinear Science and Numerical Simulation, vol. 14, no. 5, pp. 1816-1821, 2009. 
[5] Y. Cui, S. Liu, J. Tang, and Y. Meng, "Amplitude control of limit cycles in Langford system," Chaos, Solitons \& Fractals, vol. 42, no. 1, pp. 335-340, 2009.

[6] C. Du and Y. Liu, "The problem of general center-focus and bifurcation of limit cycles for a planar system of nine degrees," Journal of Computational and Applied Mathematics, vol. 223, no. 2, pp. 1043-1057, 2009.

[7] F. Li, Y. Liu, and Y. Wu, "Center conditions and bifurcation of limit cycles at three-order nilpotent critical point in a seventh degree Lyapunov system," Communications in Nonlinear Science and Numerical Simulation, vol. 16, no. 6, pp. 2598-2608, 2011.

[8] L. Mendelowitz, A. Verdugo, and R. Rand, "Dynamics of three coupled limit cycle oscillators with application to artificial intelligence," Communications in Nonlinear Science and Numerical Simulation, vol. 14, no. 1, pp. 270-283, 2009.

[9] S. M. Moghadas and B. D. Corbett, "Limit cycles in a generalized Gause-type predator-prey model," Chaos, Solitons \& Fractals, vol. 37, no. 5, pp. 1343-1355, 2008.

[10] M. Pandey, R. Rand, and A. Zehnder, "Perturbation analysis of entrainment in a micromechanical limit cycle oscillator," Communications in Nonlinear Science and Numerical Simulation, vol. 12, no. 7, pp. 1291-1301, 2007.

[11] Y.-J. Sun, "Existence and uniqueness of limit cycle for a class of nonlinear discrete-time systems," Chaos, Solitons \& Fractals, vol. 38, no. 1, pp. 89-96, 2008.

[12] D. Xiao and Z. Zhang, "On the existence and uniqueness of limit cycles for generalized Liénard systems," Journal of Mathematical Analysis and Applications, vol. 343, no. 1, pp. 299-309, 2008.

[13] P. Yu and R. Corless, "Symbolic computation of limit cycles associated with Hilbert's 16th problem," Communications in Nonlinear Science and Numerical Simulation, vol. 14, no. 12, pp. 4041-4056, 2009.

[14] R. R. V. Dodla, A. Sen, and G. L. Johnston, "Driven response of time delay coupled limit cycle oscillators," Communications in Nonlinear Science and Numerical Simulation, vol. 8, no. 3-4, pp. 493-518, 2003.

[15] V. Lakshmikantham and S. Leela, Differential and Integral Inequalities: Theory and Applications, Academic Press, New York, NY, USA, 1969. 


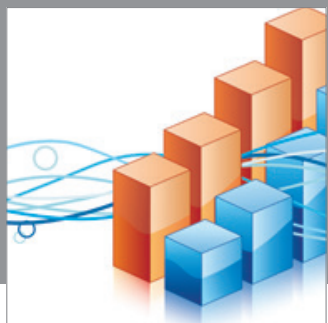

Advances in

Operations Research

mansans

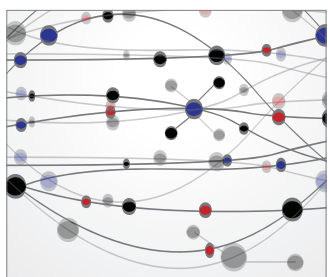

The Scientific World Journal
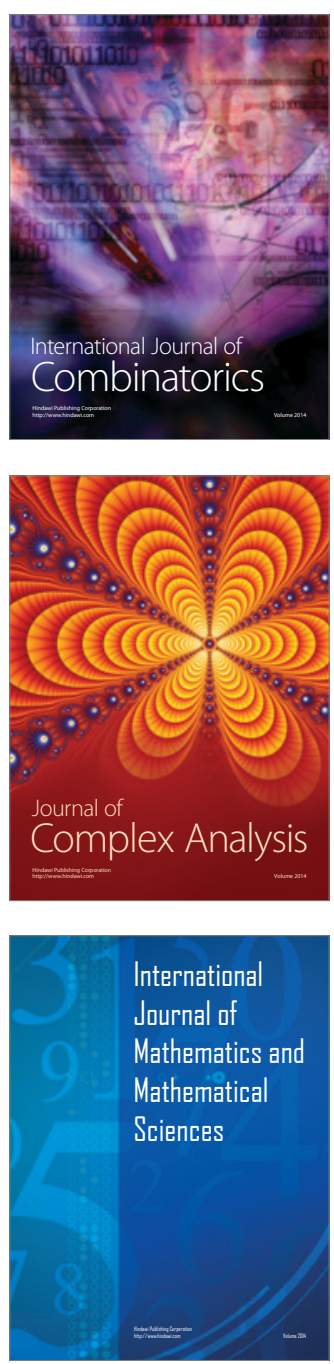
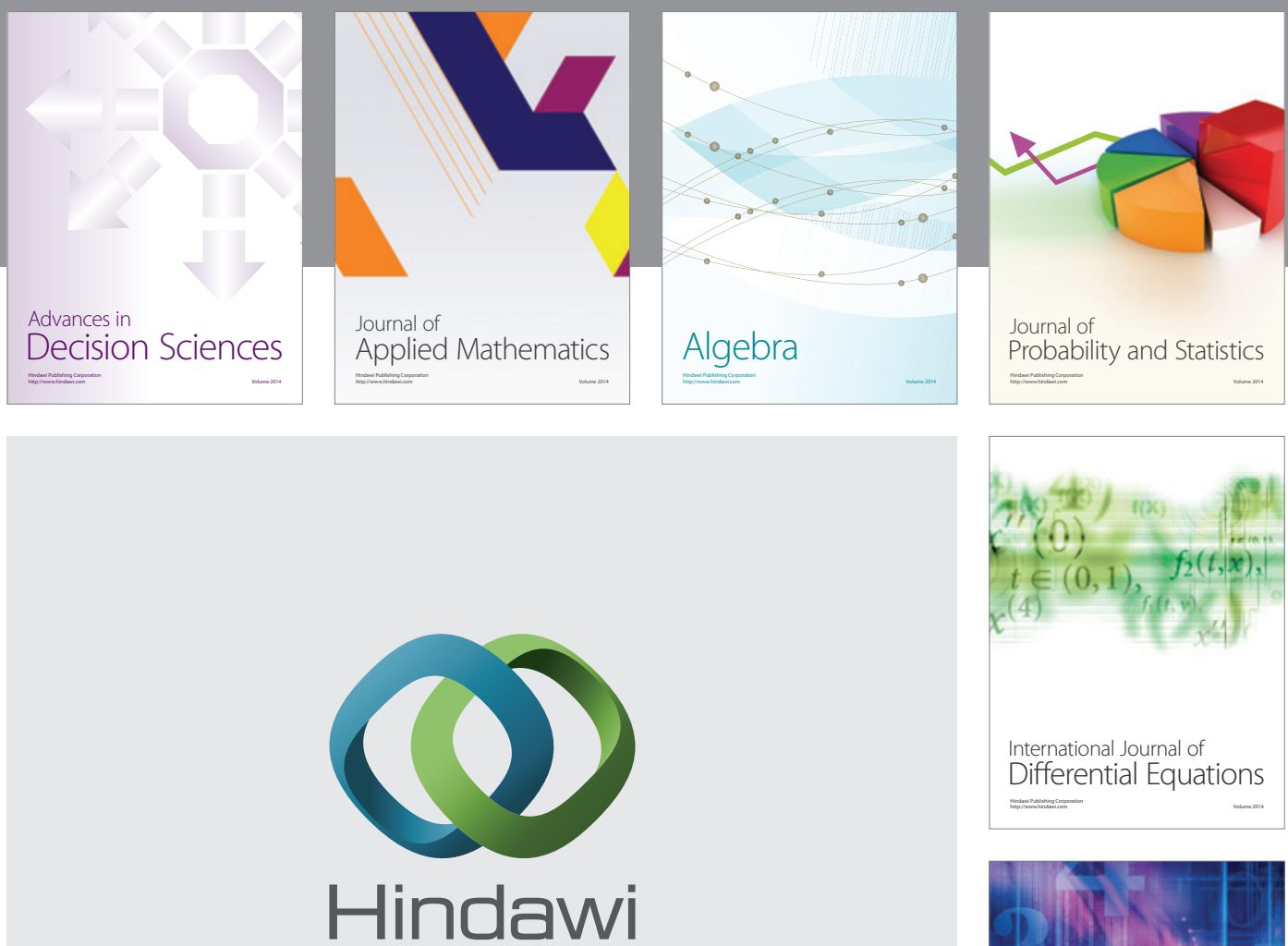

Submit your manuscripts at http://www.hindawi.com
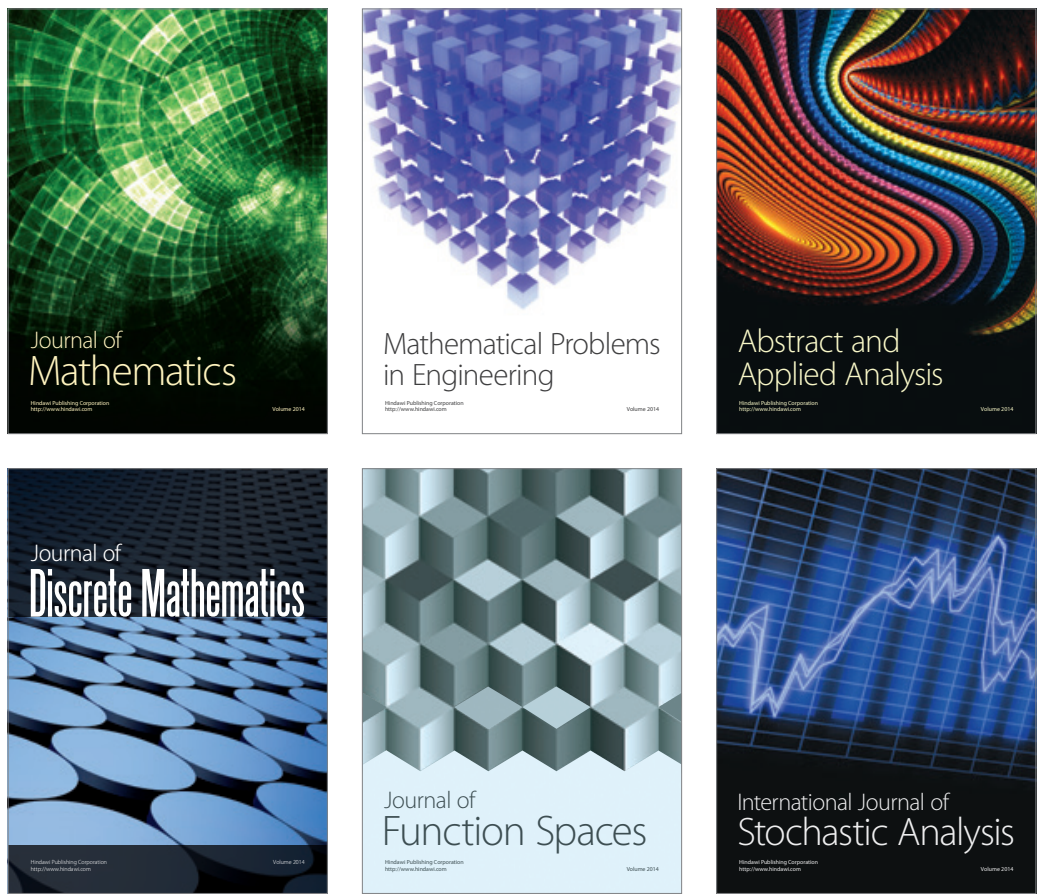

Journal of

Function Spaces

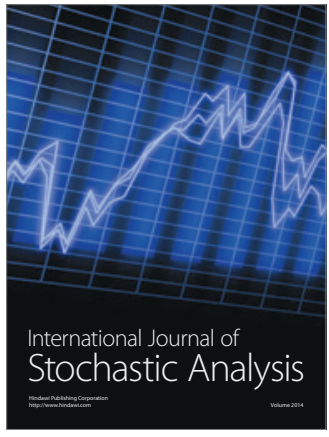

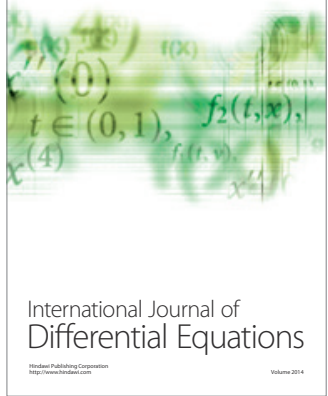
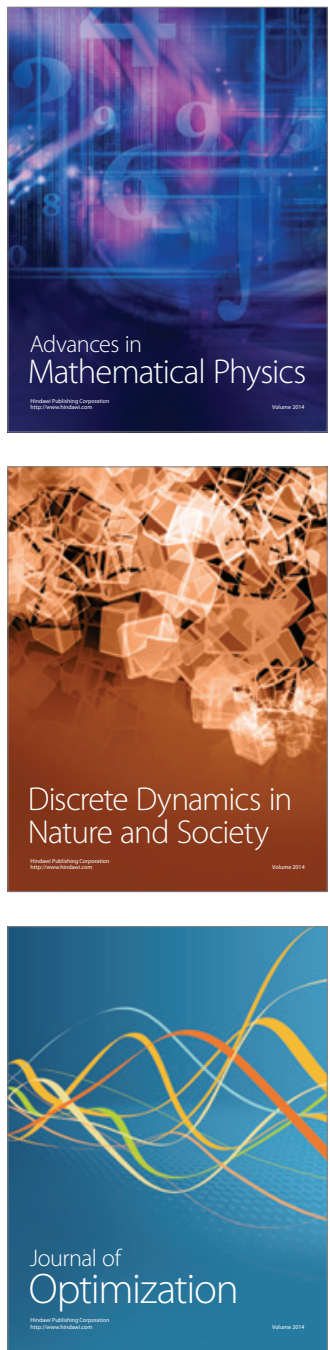